\title{
Diastolic dysfunction was predictive of all cause mortality
}

Redfield MM, Jacobsen SJ, Burnett JC Jr, et al. Burden of systolic and diastolic ventricular dysfunction in the community: appreciating the scope of the heart failure epidemic. JAMA 2003;289:194-202.

\section{QUESTION: Is mild, moderate, or severe diastolic dysfunction predictive of all cause mortality?}

Design

Cohort study with median 3.5 person years of follow up.

\section{Setting}

Olmsted County, Minnesota, USA.

\section{Participants}

2042 of 4203 eligible, invited adults who were $\geq 45$ years of age (mean age $63 \mathrm{y}$ ), resided in Olmsted County, Minnesota, and were participants in the Rochester Epidemiology Project.

\section{Assessment of risk factors}

Blood pressure, height, weight and body mass index; clinical diagnoses of hypertension, myocardial infarction, coronary artery disease, and diabetes mellitus; determination of congestive heart failure (CHF) diagnosis (validated using Framingham criteria); and preclinical diastolic or systolic dysfunction (no diagnosis of $\mathrm{CHF}$, but with either diastolic or systolic dysfunction determined by echocardiography) were measured. Systolic function was categorised by ejection fraction (EF) $\leq 50 \%$ and $\leq 40 \%$. Diastolic function was categorised as mild (impaired relaxation without increased filling pressures), moderate (impaired relaxation associated with moderate elevation of filling pressures or pseudonormal filling), or severe (advanced reduction in compliance or reversible or fixed restrictive filling). 2 Doppler criteria were required for the classification of moderate or severe diastolic dysfunction. Patients fulfilling only 1 criterion or with borderline variables for diastolic dysfunction were classified as "indeterminate."

\section{Main outcome measures}

Risk of all cause mortality associated with diastolic dysfunction.

\section{Main results}

Of 1799 patients with determined normal or abnormal diastolic function, 502 patients (28\%) had abnormal diastolic function (mild [21\%], moderate [6.6\%], and severe $[0.7 \%]$ ). 263 patients had indeterminate diastolic function. Diastolic dysfunction increased with increasing age and the presence of cardiovascular disease, diabetes, and systolic dysfunction. A greater increase in mortality occurred in patients with mild, moderate, or severe diastolic dysfunction than in patients with normal

Diastolic dysfunction as predictor of all cause mortality*

\begin{tabular}{ll} 
Variables & Hazard ratio $(95 \% \mathrm{Cl})$ \\
$\begin{array}{l}\text { Mild diastolic dysfunction } v \\
\text { normal diastolic function }\end{array}$ & $8.31(3.00$ to 23.10$)$ \\
\hline $\begin{array}{l}\text { Moderate to severe diastolic } \\
\text { dysfunction } v \text { normal } \\
\text { diastolic function }\end{array}$ & 10.17 (3.28 to 31.00$)$ \\
\hline
\end{tabular}

${ }^{*}$ Adjusted for age, sex, and ejection fraction. diastolic function (table). Overall, 123 patients (6\%) had any systolic dysfunction (EF $\leq 50 \%)$ and 40 patients $(2 \%)$ had moderate to severe systolic dysfunction ( $\mathrm{EF} \leq 40 \%$ ). Framingham validated CHF was diagnosed in 45 patients $(2.2 \%, 95 \%$ CI 1.6 to 2.8$)$.

\section{Conclusion}

Mild, moderate, or severe diastolic dysfunction was associated with increased all cause mortality.

\section{COMMENTARY}

The study by Redfield $e t$ al lends further support to the concept that diastolic dysfunction is prevalent, causes symptoms of CHF, and is associated with increased mortality. Diastolic dysfunction refers to a pathologically non-compliant left ventricle (LV) in diastole, which is the result of the combination of slowed $L V$ relaxation and increased stiffness caused by hypertension, diabetes, ischaemia, or a combination. ${ }^{1}$

The study uses a model of diastolic dysfunction that assumes temporal progression of dysfunction from mild to moderate to severe based on left heart filling patterns obtained by state of the art echo Doppler techniques. Some aspects of this approach are problematic. The temporal progression of diastolic dysfunction has not been shown, the use of echo Doppler parameters was obtained at only a single point in time, and the use of these filling patterns to reflect $\mathrm{LV}$ properties in a wide range of patients is not the standard of measurement. Although these limitations should be addressed in future research, the findings of this study are very useful.

Of interest, the prevalence of diastolic dysfunction in patients $\geq 65$ years of age was $54 \%$. This high prevalence probably resulted from inclusion of patients with age related stiffening of the LV. It may be possible to exclude these patients by age adjusting the echo Doppler criteria; however, the decision to do this would depend on whether aging is considered to be normal or pathological.

In this study, the correlation of diastolic dysfunction with mortality appropriately corrects for age, sex, and EF, but fails to control for the direct effects of hypertension and diabetes mellitus on mortality. One might expect the direct effects of hypertension and diabetes mellitus to be substantial compared with their indirect effects through diastolic dysfunction. Such an analysis would be worthwhile. Despite this limitation, the study is an important effort toward recognising the prevalence and significance of diastolic dysfunction, and particularly, the prevalence of preclinical diastolic dysfunction. This knowledge should lead to the acceleration of efforts to devise a practical treatment regimen.

Jay S Meisner, MD, PhD

Albert Einstein College of Medicine of Yeshiva University Bronx, New York, USA

1 Yellin EL, Meisner JS. Physiology of diastolic function and transmitral pressure-flow relations. Cardiol Clin 2000;18: 411-33.
Sources of funding: National Institutes of Health; Marriott Foundation; Miami Heart Research Institute; Mayo Foundation.

For correspondence: Dr M M Redfield,

Mayo Clinic, Rochester, MN, USA. redfield.margaret@ mayo.edu

Abstract and commentary also appear in ACP Journal 